\title{
VECTOR FIELDS ON CERTAIN QUOTIENTS OF COMPLEX STIEFEL MANIFOLDS
}

\author{
SHILPA GONDHALI* AND PARAMESWARAN SANKARAN**
}

\begin{abstract}
We consider quotients of complex Stiefel manifolds by finite cyclic groups whose action is induced by the scalar multiplication on the corresponding complex vector space. We obtain a description of their tangent bundles, compute their mod $p$ cohomology and obtain estimates for their span (with respect to their standard differentiable structure). We compute the Pontrjagin and Stiefel-Whitney classes of these manifolds and give applications to their stable parallelizability.
\end{abstract}

\section{INTRODUCTION}

Let $W_{n, k}, 1 \leq k<n$, denote the complex Stiefel manifold of unitary $k$-frames $\left(v_{1}, \ldots, v_{k}\right)$ in $\mathbb{C}^{n}$ where it is understood that $\mathbb{C}^{n}$ has the standard hermitian metric. One has the identification $W_{n, k}=U(n) / U(n-k)$ where $U(n)$ denotes the group of unitary transformations of $\mathbb{C}^{n}$ and $U(n-k)$ is imbedded in $U(n)$ as the subgroup that fixes the first $k$ standard basis vectors $e_{1}, \ldots, e_{k} \in \mathbb{C}^{n}$.

One also has the complex projective Stiefel manifold $P W_{n, k}$ defined as the quotient of $W_{n, k}$ modulo the free action of the circle group $\mathbb{S}^{1}$ which acts via scalar multiplication: $z\left(v_{1}, \ldots, v_{k}\right)=\left(z v_{1}, \ldots, z v_{k}\right)$ for $\left(v_{1}, \ldots, v_{k}\right) \in W_{n, k}$ and $z \in \mathbb{S}^{1}$. Note that $P W_{n, k}=U(n) /\left(\mathbb{S}^{1} \times U(n-k)\right)$ where $\mathbb{S}^{1}=\{z \in \mathbb{C}|| z \mid=1\}$ is identified with the centre of $U(n)$. Observe that $\mathbb{S}^{1} \times U(n-k)=U(1) \times$ $U(n-k) \subset U(k) \times U(n-k) \subset U(n)$ where $U(1) \subset U(k)$ is the centre of $U(k)$ and $U(k) \times U(n-k)$ is the subgroup of $U(n)$ that stabilizes the complex vector subspace $\mathbb{C}^{k}$, spanned by $e_{1}, \ldots, e_{k}$. Thus we get an equivalent description $P W_{n, k}=U(n) /(U(1) \times U(n-k))$.

2010 Mathematics Subject Classification. 57R25.

Key words and phrases. $m$-projective Stiefel manifolds, span, stable span, parallelizability, cohomology, characteristic classes. 
We define $W_{n, k ; m}$ to be the quotient of $W_{n, k}$ by the subgroup $\Gamma_{m} \subset \mathbb{S}^{1}$ of $m$-th roots of unity. Thus $\operatorname{dim} W_{n, k ; m}=\operatorname{dim} W_{n, k}=k(2 n-k)$. The manifolds $W_{n, k ; m}$ will be referred to as the m-projective Stiefel manifolds. Clearly $W_{n, k ; m}$ is the coset space $U(n) /\left(\Gamma_{m} \times U(n-k)\right)$ and the obvious quotient map $W_{n, k ; m} \longrightarrow$ $P W_{n, k}$ is the projection of a principal bundle with fibre and structure group $\mathbb{S}^{1} / \Gamma_{m} \cong \mathbb{S}^{1}$. Also the projection $W_{n, k} \longrightarrow W_{n, k ; m}$ is a covering map with deck transformation group $\Gamma_{m}$. In particular $\pi_{1}\left(W_{n, k ; m}\right) \cong \Gamma_{m}$ and the Euler characteristic $\chi\left(W_{n, k ; m}\right)$ vanishes. The manifold $W_{n, k ; m}$ is orientable since $\Gamma_{m}$ is a subgroup of the connected group $\mathbb{S}^{1}$ which reserves the orientation on $W_{n, k}$. Our aim in this paper is to initiate the study of the topology of $W_{n, k ; m}$. In $\S 2$ we describe their tangent bundle and give (in Theorem 2.4) estimates for their span and stable span. Span and other related notions will be recalled in $\S 2$; see also [10]. We compute, in $\S 3$, the $\bmod p$ cohomology of $W_{n, k ; m}$. We also determine the height of the generator of $H^{2}\left(W_{n, k ; m} ; \mathbb{Z}\right) \cong \mathbb{Z}_{m}$. We show that, given $n, k$ where $1 \leq k<n-1, W_{n, k ; m}$ is not stably parallelizable for all but finitely many values of $m$. See Theorem 3.5 for the precise statement. When $k=n-1, W_{n, n-1 ; m}$ is parallelizable, since $W_{n, n-1} \cong S U(n-1)$.

The case $k=1$ corresponds to the (standard) lens space $L^{n}(m)=\mathbb{S}^{2 n-1} / \Gamma_{m}$. The non-parallelizability of spheres $W_{n, 1}=\mathbb{S}^{2 n-1}, n \neq 1,2$, 4 , already implies non-parallelizability of the lens spaces $L^{n}(m)$ for any $m$. Kambe's [9, §4] result on immersion dimension for $L^{n}(p), p$ an odd prime, and the fact that $L^{n}(2)=$ $\mathbb{R} P^{2 n-1}$ are not stably parallelizable except when $n=1,2,4$, implies that 'most' of $L^{n}(m), m>1$, are not stably parallelizable. From the celebrated work of Adams, we know that $\operatorname{span}\left(L^{n}(m)\right) \leq \operatorname{span}\left(\mathbb{S}^{2 n-1}\right)=\rho(2 n)-1$, where $\rho(n)$ is the Radon-Hurwitz number, defined as $\rho\left((2 c+1) 2^{4 a+b}\right)=8 a+2^{b}$, where $a, c \geq 0$ and $0 \leq b \leq 3$. See also $[8]$ for lower bounds for span of lens spaces.

In view of this, we assume that $1<k<n$ leaving out the case of lens spaces from consideration for the most part.

Our proofs involve standard arguments making use of well-known results and techniques. The description of the tangent bundle of $W_{n, k ; m}$ relies on the description of the tangent bundle of $P W_{n, k}$ due to Lam [13. Estimates for (stable) span involve well-known arguments such as those employed in the context of real 
projective Stiefel manifolds; see [10, 11]. The cohomology calculations involve spectral sequences and known results concerning the cohomology of Stiefel manifolds and of projective Stiefel manifolds (see 4] and 2]).

\section{The TANGENT BUNDLE OF $W_{n, k ; m}$}

We describe below certain canonical vector bundles over the manifold $W_{n, k ; m}$ and establish relations among them. We shall describe its tangent bundle and obtain lower bounds for their span and stable span.

Let $1 \leq k<n$ and let $m \geq 2$. Let $\Gamma_{m} \subset U(1)$ denote the group of $m$-th roots of unity. Let $\pi_{m}: W_{n, k ; m} \longrightarrow P W_{n, k}$ and $\pi_{1}: W_{n, k} \longrightarrow P W_{n, k}$ be the canonical quotient maps. These are projections of principal bundles with structure groups $U(1) / \Gamma_{m}$ and $U(1)$ respectively. Let $p_{m}: W_{n, k} \longrightarrow W_{n, k ; m}$ be the quotient map which is the universal covering projection with deck transformation group $\Gamma_{m}$. One also has the obvious covering projections $p_{m, l}: W_{n, k ; l} \longrightarrow W_{n, k ; m}$ whenever $l \mid m$. Note that $\pi_{1}=\pi_{m} \circ p_{m}$ and $p_{m}=p_{m, l} \circ p_{l}$. We shall denote by $\left[v_{1}, \ldots, v_{k}\right]_{m}$ (or simply $\left[v_{1}, \ldots, v_{k}\right]$ when there is no danger of confusion) the element $\pi_{m}\left(v_{1}, \ldots, v_{k}\right) \in W_{n, k ; m}$ where $\left(v_{1}, \ldots, v_{k}\right) \in W_{n, k}$. Also, $\pi_{1}\left(v_{1}, \ldots, v_{k}\right) \in P W_{n, k}$ will be denoted $\left[v_{1}, \ldots, v_{k}\right]_{0}$ (or more briefly $\left[v_{1}, \ldots, v_{k}\right]$ ).

Let $\zeta_{n, k}$ denote the complex line bundle over $P W_{n, k}$ associated to the principal $U(1)$-bundle $\pi_{1}: W_{n, k} \longrightarrow P W_{n, k}$. Thus, the total space of $\zeta_{n, k}$ is the fibre product $W_{n, k} \times_{U(1)} \mathbb{C}$. It is isomorphic to the bundle over $P W_{n, k}$ whose fibre over a point $\left[v_{1}, \ldots, v_{k}\right]_{0}$ is the complex vector space $\mathbb{C} v_{1} \subset \mathbb{C}^{n}$. Define $\xi_{n, k ; m}:=\pi_{m}^{*}\left(\zeta_{n, k}\right)$ and let $\gamma_{n, k ; m}$ be the complex line bundle associated to the principal $U(1)$-bundle obtained by extension of structure group via the character $\Gamma_{m} \subset U(1)$ of the $\Gamma_{m}$-bundle $W_{n, k} \longrightarrow W_{n, k ; m}$. (When $m=2$, $\xi_{n, k ; m}$ is the complexification of the real line bundle associated to the double cover $W_{n, k} \longrightarrow W_{n, k ; 2}$.) Explicitly, $\gamma_{n, k ; m}$ has total space the twisted product $W_{n, k} \times_{\Gamma_{m}} \mathbb{C}$ where $\Gamma_{m}$ operates on $\mathbb{C}$ by scalar multiplication. We have the following lemma. We outline a proof, which is elementary, as the lemma will be 
used throughout. For any vector bundle $\eta, \eta^{l}$ denotes the $l$-fold tensor product with itself and $l \eta$, the $l$-fold Whitney sum with itself.

Lemma 2.1. (i) The complex line bundle associated to the principal $U(1) / \Gamma_{m} \cong$ $U(1)$-bundle with projection $W_{n, k ; m} \longrightarrow P W_{n, k}$ is isomorphic to $\zeta_{n, k}^{m}$.

(ii) One has an isomorphism $\xi_{n, k ; m} \cong \gamma_{n, k ; m}$ of complex line bundles over $W_{n, k ; m}$.

Proof. (i) The isomorphism $U(1) / \Gamma_{m} \longrightarrow U(1)$ is induced by the homomorphism $z \mapsto z^{m}$ of $U(1)$ onto itself. This homomorphism induces the map $\eta \mapsto \eta^{m}$, for any line bundle $\eta$ associated to a principal $U(1)$-bundle. By definition, $\zeta_{n, k}$ is associated to the principal $U(1)$-bundle $\pi_{1}: W_{n, k} \longrightarrow P W_{n, k}$. Since $\pi_{m}: W_{n, k ; m} \longrightarrow P W_{n, k}$ is the $U(1) / \Gamma_{m}$-bundle associated to $\pi_{1}$, it follows that the complex line bundle associated to the principal $U(1) / \Gamma_{m} \cong U(1)$-bundle $\pi_{m}$ is $\zeta_{n, k}^{m}$.

(ii) By the very definition of $\gamma_{n, k ; m}$, its total space has the description $E\left(\gamma_{n, k ; m}\right)=$ $\left\{[x, z] \mid x \in W_{n, k}, z \in \mathbb{C}\right\}$ where $[x, z]=\left[x^{\prime}, z^{\prime}\right]$ if and only if $x g=x^{\prime}, g^{-1} z=$ $z^{\prime}$ for some $g \in \Gamma_{m}$. Also, one has $E\left(\xi_{n, k}\right)=\left\{\left(p_{m}(v), t v_{1}\right) \mid t \in \mathbb{C}, x=\right.$ $\left.\left(v_{1}, \ldots, v_{k}\right) \in W_{n, k}\right\}$. Consider the map $f: E\left(\xi_{n, k ; m}\right) \longrightarrow E\left(\gamma_{n, k ; m}\right)$ defined as $\left(p_{m}(v), t v_{1}\right) \mapsto[v ; t]$. It is readily checked that this is a well-defined continuous map that covers the identity map of the base space $W_{n, k ; m}$, and is a linear isomorphism on each fibre. This completes the proof.

Observe that $\xi_{n, k ; m}^{l}$, which corresponds to the character $\Gamma_{m} \longrightarrow U(1), z \mapsto z^{l}$, is non-trivial when $1 \leq l<m$. In particular it follows that the order of the class of $\xi_{n, k ; m}$ in the Picard group $\operatorname{Pic}\left(W_{n, k ; m}\right)$ of $W_{n, k ; m}$ is $m$. Indeed, $\xi_{n, k ; m}$ is a generator of $\operatorname{Pic}\left(W_{n, k ; m}\right) \cong \mathbb{Z}_{m}$. For, one has $H_{1}\left(W_{n, k ; m} ; \mathbb{Z}\right) \cong$ $\pi_{1}\left(W_{n, k ; m}\right) \cong \mathbb{Z}_{m}$. It is not difficult to see that $H_{2}\left(W_{n, k ; m} ; \mathbb{Z}\right)=0$. Hence $\operatorname{Pic}\left(W_{n, k ; m}\right) \cong H^{2}\left(W_{n, k ; m} ; \mathbb{Z}\right) \cong \mathbb{Z}_{m}$ by the universal coefficient theorem. The projection $\pi_{m}: W_{n, k ; m} \longrightarrow P W_{n, k}$ induces a surjection $H^{2}\left(P W_{n, k} ; \mathbb{Z}\right) \cong \mathbb{Z} \longrightarrow$ $\mathbb{Z}_{m} \cong H^{2}\left(W_{n, k ; m} ; \mathbb{Z}\right)$ and hence maps the generator $c_{1}\left(\zeta_{n, k}\right)$ to the generator of $\mathbb{Z}_{m}$. By the naturality of Chern classes we see that $c_{1}\left(\xi_{n, k ; m}\right)$ is a generator of $H^{2}\left(W_{n, k ; m} ; \mathbb{Z}\right) \cong \mathbb{Z}_{m}$. Summarising we have 
Lemma 2.2. The Picard group $\operatorname{Pic}\left(W_{n, k ; m}\right)$ is isomorphic to $\mathbb{Z}_{m}$ and is generated by $\xi_{n, k ; m}$.

The following isomorphism of complex vector bundles on $P W_{n, k}$ is well-known and is due to K.-Y. Lam [13]: $k \zeta_{n, k} \oplus \beta_{n, k} \cong n \varepsilon_{\mathbb{C}}$ where $\varepsilon_{\mathbb{C}}$ denotes the trivial complex line bundle and $\beta_{n, k}$ is the complex $(n-k)$-plane bundle whose fibre over $\left[v_{1}, \ldots, v_{k}\right]_{0}$ is the vector space $\left\{v_{1}, \ldots, v_{k}\right\}^{\perp} \subset \mathbb{C}^{n}$ where the orthogonal complement is taken with respect to the standard hermitian inner product on $\mathbb{C}^{n}$. Pulling back to $W_{n, k ; m}$ via the projection $\pi_{n, k ; m}$ we obtain an isomorphism

$$
k \xi_{n, k ; m} \oplus \beta_{n, k ; m} \cong n \varepsilon_{\mathbb{C}}
$$

of complex vector bundles over $W_{n, k ; m}$ where $\beta_{n, k ; m}:=\pi_{m}^{*}\left(\beta_{n, k}\right)$. Tensoring with the dual bundle $\xi_{n, k ; m}^{\vee} \cong \xi_{n, k ; m}^{m-1}$ we get $k \varepsilon_{\mathbb{C}} \oplus \xi_{n, k ; m}^{\vee} \otimes_{\mathbb{C}} \beta_{n, k ; m} \cong n \xi_{n, k ; m}^{\vee}=$ $n \xi_{n, k ; m}^{m-1}$. Taking duals, we obtain

$$
k \varepsilon_{\mathbb{C}} \oplus \xi_{n, k ; m} \otimes_{\mathbb{C}} \beta_{n, k ; m}^{\vee} \cong n \xi_{n, k ; m} .
$$

Recall from [13, Theorem 3.2] that the tangent bundle $\tau P W_{n, k}$ of $P W_{n, k}$ is isomorphic to the (real) vector bundle $k \zeta_{n, k}^{\vee} \otimes_{\mathbb{C}} \beta_{n, k} \oplus\left(k^{2}-1\right) \varepsilon_{\mathbb{R}}$. Since $\pi_{m}: W_{n, k ; m} \longrightarrow P W_{n, k}$ is a principal $\mathbb{S}^{1}$-bundle, we have

$$
\tau W_{n, k ; m} \cong k \xi_{n, k ; m}^{\vee} \otimes_{\mathbb{C}} \beta_{n, k ; m} \oplus k^{2} \varepsilon_{\mathbb{R}} .
$$

In the above isomorphism, and in the sequel, we have used the same symbol to denote a complex vector bundle and its underlying real vector bundle, as there is no risk of confusion.

Remark 2.3. Assume that $k$ is even, equivalently $W_{n, k ; m}$ is even dimensional. Then $\tau W_{n, k ; m}$ has a complex structure arising from the isomorphism of vector bundles given in (3). Thus $W_{n, k ; m}$ admits an almost complex structure. Recall that, by the work of Wang [17, $W_{n, k}=S U(n) / S U(n-k)$ admits a complex structure invariant under the left action of $S U(n)$. When $m$ divides $n, \Gamma_{m}$ is contained in the centre $\Gamma_{n}$ of $S U(n)$. In this case the action of $\Gamma_{m}$ on $W_{n, k}$ preserves the complex structure. We conclude that $W_{n, k ; m}$ admits a complex structure when it is even dimensional and $m \mid n$. 
Using the isomorphism (1) and the fact that $\varepsilon_{\mathbb{C}}=2 \varepsilon_{\mathbb{R}}$, we obtain an isomorphism

$$
\tau W_{n, k ; m} \oplus k^{2} \varepsilon_{\mathbb{R}} \cong k\left(\xi_{n, k ; m}^{\vee} \otimes_{\mathbb{C}} \beta_{n, k ; m} \oplus k \varepsilon_{\mathbb{C}}\right)=n k \xi_{n, k ; m}^{\vee}
$$

of real vector bundles.

Recall that the span of a smooth manifold $M$ is the maximum number $r \geq 0$ for which there exist $r$ everywhere linearly independent vector fields on $M$. Equivalently span of $M$ is the maximum number $r$ such that $\tau M \cong r \varepsilon_{\mathbb{R}} \oplus \eta$ for some vector bundle $\eta$. The stable span of $M$ is the maximum number $s$ such that $\tau M \oplus t \varepsilon_{\mathbb{R}} \cong(s+t) \varepsilon_{\mathbb{R}} \oplus \theta$ for some vector bundle $\theta$ where $t>0$. Indeed one may take $t=1$ in the above definition of stable span. The rank of $\theta$ is then called the geometric dimension of $\tau M$. We denote the span of $M$ by $\operatorname{span}(M)$. The notions of span, stable span, and geometric dimension can be extended in an obvious manner to any vector bundle. The reader may refer to [10] and [1] for a detailed discussion on the vector field problem, which asks for the determination of the span of a given smooth manifold.

Theorem 2.4. Suppose that $2 \leq k<n$ and $m \geq 2$. Then:

(i) $\operatorname{span}\left(W_{n, k ; m}\right)>$ stable $\operatorname{span}\left(P W_{n, k}\right) \geq \operatorname{dim}\left(W_{n, k ; m}\right)-2 n+1$. Moreover, when $n$ is even, $\operatorname{span}\left(W_{n, k ; m}\right)>\operatorname{dim}\left(W_{n, k ; m}\right)-2 n+3$.

(ii) $\operatorname{span}\left(W_{n, k ; m}\right)>\operatorname{stable} \operatorname{span}\left(W_{n, k-1 ; m}\right)$.

(iii) $W_{n, n-1 ; m}$ is parallelizable.

Proof. (i) Since $\pi_{m}$ is a principal $\mathbb{S}^{1}$-bundle, one has the bundle isomorphism $\tau\left(W_{n, k ; m}\right) \cong \pi^{*}\left(\tau P W_{n, k}\right) \oplus \varepsilon_{\mathbb{R}}=\pi^{*}\left(\tau P W_{n, k} \oplus \varepsilon_{\mathbb{R}}\right)$. Hence $\operatorname{span}\left(W_{n, k ; m}\right)>$ stable $\operatorname{span}\left(P W_{n, k}\right)$. Now consider the projection $q: P W_{n, k} \longrightarrow \mathbb{C} P^{n-1}$ defined as $\left[v_{1}, \ldots, v_{k}\right] \mapsto\left[v_{1}\right]$. The stable tangent bundle $\tau P W_{n, k} \oplus\left(k^{2}+1\right) \varepsilon_{\mathbb{R}}$ is isomorphic to $n k \zeta_{n, k}=q^{*}\left(n k \zeta_{n, 1}\right)$. Clearly the bundle $n k \zeta_{n, 1}$ over $\mathbb{C} P^{n-1}$ contains a trivial real vector bundle of rank $2(n k-(n-1))$. (See [7.) Therefore the stable span of $P W_{n, k}$ is at least $2 n k-2(n-1)-\left(k^{2}+1\right)=\operatorname{dim} W_{n, k ; m}-2 n+1$.

Let $n$ be even. The complex 2-plane bundle $\zeta_{n, 1} \oplus \zeta_{n, 1}^{\vee}$ evidently admits a reduction of structure group to $S U(2)=S p(1)$. Hence it is the underlying complex vector bundle of a quaternionic line bundle. Any such bundle can be classified by a map into the quaternionic projective space $\mathbb{H} P^{r}$ where 
$r=\left\lfloor(1 / 4) \operatorname{dim}_{\mathbb{R}}\left(\mathbb{C} P^{n-1}\right)\right\rfloor=n / 2-1$. That is, there exists a continuous map $h: \mathbb{C} P^{n-1} \longrightarrow \mathbb{H} P^{r}$ such that $h^{*}(\omega) \cong \zeta_{n, 1} \oplus \zeta_{n, 1}^{\vee}$ where $\omega$ is the canonical quaternionic line bundle over $\mathbb{H} P^{r}$. The underlying real vector bundle $(n k / 2) \omega$ admits $(2 n k-4 r) \varepsilon_{\mathbb{R}}$ as a summand and so, working with underlying real vector bundles throughout, we have $n k \zeta_{n, 1}=(n k / 2)\left(q^{*}\left(\zeta_{n, 1} \oplus \zeta_{n, 1}^{\vee}\right)\right)=h^{*}((n k / 2) \cdot \omega)=$ $(2 n k-4 r) \varepsilon_{\mathbb{R}} \oplus \eta$ for some real vector bundle $\eta$. As before, it follows that $\operatorname{span}\left(W_{n, k ; m}\right)>$ stable $\operatorname{span}\left(P W_{n, k}\right) \geq \operatorname{dim} W_{n, k ; m}-2 n+3$.

(ii) Consider the fibre bundle projection $W_{n, k} \longrightarrow W_{n, k-1}$ with fibre $\mathbb{S}^{2 n-2 k+1}$. Since it is $\Gamma_{m}$-equivariant, we obtain a $\mathbb{S}^{2 n-2 k+1}$-bundle with projection $p$ : $W_{n, k ; m} \longrightarrow W_{n, k-1 ; m}$. Note that $p^{*}\left(\xi_{n, k-1 ; m}\right)=\xi_{n, k ; m}$ and $p^{*}\left(\beta_{n, k-1 ; m}\right)=$ $\beta_{n, k ; m} \oplus \xi_{n, k ; m}$. Write $k^{2} \varepsilon_{\mathbb{R}}$ as $(k-1)^{2} \varepsilon_{\mathbb{R}} \oplus(k-1)\left(\xi_{n, k ; m}^{\vee} \otimes_{\mathbb{C}} \xi_{n, k ; m}\right) \oplus \varepsilon_{\mathbb{R}}$. Substituting this in the expression (3) for $\tau W_{n, k ; m}$ and observing that $k \xi_{n, k ; m}^{\vee} \otimes \beta_{n, k ; m} \oplus$ $(k-1) \xi_{n, k ; m}^{\vee} \otimes_{\mathbb{C}} \xi_{n, k ; m}=(k-1) \xi_{n, k ; m}^{\vee} \otimes\left(\beta_{n, k ; m} \oplus \xi_{n, k ; m}\right) \oplus \xi_{n, k ; m}^{\vee} \otimes \beta_{n, k ; m}$, we obtain that $\tau W_{n, k ; m} \cong p^{*}\left(\tau W_{n, k ; m-1}\right) \oplus \varepsilon_{\mathbb{R}} \oplus \xi_{n, k ; m}^{\vee} \otimes \beta_{n, k ; m} \cong p^{*}\left(\tau W_{n, k ; m-1} \oplus\right.$ $\left.\varepsilon_{\mathbb{R}}\right) \oplus \xi_{n, k ; m}^{\vee} \otimes \beta_{n, k ; m}$. Therefore $\operatorname{span}\left(W_{n, k ; m}\right) \geq \operatorname{span}\left(\tau W_{n, k-1 ; m} \oplus \varepsilon_{\mathbb{R}}\right)>$ stable $\operatorname{span}\left(W_{n, k-1 ; m}\right)$ as asserted.

(iii) Note that $W_{n, n-1} \cong S U(n)$. Therefore $W_{n, n-1 ; m}$, being a quotient of a Lie group by a finite subgroup, is parallelizable.

We refer the reader to [18] and $[8$ for the span of lens spaces.

Proposition 2.5. Let $2 \leq k<n$ and let $m \geq 2$. One has

$$
\operatorname{span}\left(W_{n, k ; m}\right)=\operatorname{stable} \operatorname{span}\left(W_{n, k ; m}\right)
$$

in each of the following cases: (i) $k$ is even, (ii) $n$ is odd, and, (iii) $n \equiv 2$ $\bmod 4$.

Proof. From (3) we obtain that $\operatorname{span}\left(W_{n, k ; m}\right) \geq k^{2} \geq 4$. Since $W_{n, k ; m}$ is orientable, the first Stiefel-Whitney class $w_{1}\left(W_{n, k ; m}\right)$ vanishes. As observed already in the introduction, the Euler characteristic $\chi\left(W_{n, k ; m}\right)$ vanishes. Furthermore, it follows from (4) that the Stiefel-Whitney classes $w_{1}\left(W_{n, k ; m}\right), w_{2}\left(W_{n, k ; m}\right)$ vanish when $n k$ is even. (We shall give a formula for the total Stiefel-Whitney class of $W_{n, k ; m}$ in Proposition 3.4 which also implies that $w_{i}\left(W_{n, k ; m}\right)=0, i=1,2$ when $n k$ is even.) 
Our hypotheses on $k$ and $n$ imply that $d=\operatorname{dim}\left(W_{n, k ; m}\right)=k(2 n-k)$ is even when $k$ is even, $d \equiv 1 \bmod 4$ when both $k$ and $n$ are odd, and $d \equiv 3 \bmod 8$ when $k$ is odd and $n \equiv 2 \bmod 4$. The proposition now follows from the work of Koschorke. More precisely, (i) follows from [12, Theorem 20.1] and, assuming, as we may, that $k$ is odd, (ii) and (iii) follow, respectively, from Corollaries 20.9, and 20.10 of $[12$.

Remark 2.6. Recall that the generalized vector field problem asks for the determination of the geometric dimension of multiples of the Hopf bundle $\xi_{n}$ over the real projective space $\mathbb{R} P^{n}$. When $m=2, W_{n, 1 ; 2}=\mathbb{R} P^{2 n-1}$ and the bundle $\xi_{n, 1 ; 2}$ is isomorphic, as a real vector bundle, to $2 \xi_{n-1}$. Denoting by $p: W_{n, k ; 2} \longrightarrow \mathbb{R} P^{2 n-1}$ the projection $\left[v_{1}, \ldots, v_{k}\right] \mapsto\left[v_{1}\right]$ we see that $\xi_{n, k ; 2} \cong p^{*}\left(2 \xi_{2 n-1}\right)$. Therefore, using the bundle isomorphism (4), we have

$$
\text { stable } \operatorname{span}\left(W_{n, k ; 2}\right) \geq \operatorname{span}\left(2 n k \xi_{2 n-1}\right)-k^{2} .
$$

Invoking Proposition 2.5 we obtain the following lower bound:

$$
\operatorname{span}\left(W_{n, k ; 2}\right) \geq \operatorname{span}\left(2 n k \xi_{2 n-1}\right)-k^{2}
$$

when $k$ is even, or $n$ is odd, or $n \equiv 2 \bmod 4$. Although the generalized vector field problem is yet to be solved completely, the precise value of the span of $r \xi_{n}$ is known from the work of Lam [14, Theorem 1.1] when $r=8 l+p, n=8 m+q$, $l \geq m \geq 0,\left(\begin{array}{c}l \\ m\end{array}\right)$ is odd, $0 \leq p, q \leq 7$. See also [15]. In many cases, (6) yields a better lower bound than Theorem 2.4(i).

We conclude this section with the following

Proposition 2.7. Let $m \geq 2$ be an integer. Let $X$ be any topological space and let $\xi$ be a complex line bundle over $X$ such that (i) $\xi$ admits a reduction of structure group to $\mathbb{Z}_{m}$, and, (ii) $n \xi$ admits $k$ everywhere $\mathbb{C}$-linearly independent cross-sections. Then there exists a continuous map $f: X \longrightarrow W_{n, k ; m}$ such that $f^{*}\left(\xi_{n, k ; m}\right) \cong \xi$.

Proof. Let $p: \widetilde{X} \longrightarrow X$ be a regular covering projection with deck transformation group $\Gamma_{m} \cong \mathbb{Z}_{m}$ such that $\xi$ is isomorphic to the bundle with projection $E:=\widetilde{X} \times_{\Gamma_{m}} \mathbb{C} \longrightarrow X$ where $\Gamma_{m}$ acts on $\mathbb{C}$ via a character $\Gamma_{m} \longrightarrow \mathbb{C}^{*}$. The 
existence of such a covering is the content of (i). We identify the total space of $\xi$ with $E$. Observe that $\xi$ admits a hermitian metric: $\left(e, e^{\prime}\right) \mapsto z \bar{z}^{\prime}$ is a hermitian metric where $e=[x, z], e^{\prime}=\left[x, z^{\prime}\right] \in E, x \in X, z, z^{\prime} \in \mathbb{C}$. Consequently $\xi^{\vee}$ also admits a hermitian metric.

In view of (ii) and the existence of a hermitian metric on $n \xi$, we have a splitting $n \xi \cong k \varepsilon_{\mathbb{C}} \oplus \theta$. Taking duals, we get $n \xi^{\vee} \cong k \varepsilon_{\mathbb{C}} \oplus \theta^{\vee}$. Tensoring with $\xi$, we see that $n \varepsilon_{\mathbb{C}}=k \xi \oplus \eta$ where $\eta:=\xi \otimes \theta^{\vee}$. Then $\eta$ also admits a hermitian metric which is such that each copy of $\xi$ and $\eta$ are pairwise orthogonal.

For any hermitian vector bundle $\nu$ of rank $n$ over $X$, one has an associated $W_{n, k ; m}$-bundle, denoted $W_{n, k ; m}(\nu)$, defined as the space of all $\Gamma_{m}$-equivalence classes of unitary $k$-frames in each fibre of $\nu$. When $\nu$ is trivial, this is just the product bundle $X \times W_{n, k ; m} \longrightarrow X$.

Now one has a cross-section $\sigma: X \longrightarrow X \times W_{n, k ; m}=W_{n, k ; m}(k \xi \oplus \eta)$ defined as follows: For any $x \in X$, let $\widetilde{x} \in p^{-1}(x)$ be any point in the fibre over $x \in X$. We identify $\widetilde{x}$ with $[\widetilde{x}, 1] \in \widetilde{X} \times_{\Gamma_{m}} \mathbb{C}=E(\xi)$. Then $\sigma(x)=[\widetilde{x}, \ldots, \widetilde{x} ; 0] \in W_{n, k ; m}(k \xi \oplus$

$\eta)$ is well-defined and is independent of the choice of $\widetilde{x}$ in $p^{-1}(x)$. Since $p: \widetilde{X} \longrightarrow$ $X$, is a local homeomorphism, it is immediate that $\sigma$ is continuous.

The desired map $f: X \longrightarrow W_{n, k}$ is now obtained as the composition $p r_{2} \circ$ $\sigma$.

Remark 2.8. An analogue of the above property for real projective Stiefel manifolds was established in [3]. A similar universal property for complex projective Stiefel manifolds was established in [2], under the additional assumption that $X$ be a finite CW complex.

\section{The Mod $p$ COHOMOLOGY}

In this section we shall describe the mod $p$ cohomology of $W_{n, k ; m}$ where $p$ is a prime. Recall that $H^{*}\left(W_{n, k} ; \mathbb{Z}\right)$ is isomorphic to the exterior algebra $\Lambda_{\mathbb{Z}}\left(v_{2 n-2 k+1}, \ldots, v_{2 n-1}\right)$ where $v_{q} \in H^{q}\left(W_{n, k} ; \mathbb{Z}\right)$. This result is attributed to C. Ehresmann by Borel [4, Prop. 9.1]. It is customary to denote by $\Lambda_{\mathbb{Z}_{p}}\left(x_{1}, \ldots, x_{k}\right)$ any graded commutative algebra $A$ over $\mathbb{Z}_{p}$ in which square-free monomials in $x_{1}, \ldots, x_{r}$ form a basis. (If $p$ is odd, and all the generators $x_{j}$ have odd degree, 
then $A$ is isomorphic to the exterior algebra. However when $p=2$, it need not be so.) This convention will be used in what follows.

Notations: Let $N:=2 N^{\prime}$ where $N^{\prime}:=N_{p}^{\prime}=\min _{n-k+1 \leq j \leq n}\left\{j \mid\left(\begin{array}{c}n \\ j\end{array}\right) \not \equiv 0\right.$ $\bmod p$. (Note that the value of $N^{\prime}$ depends on $n, k$ and $p$.) In what follows, we shall label (homogeneous) generators of a graded algebra by their degrees. Thus $\left|y_{j}\right|=j$ when $y_{j} \in H^{*}(X ; R)$.

Theorem 3.1. Suppose that $2 \leq k<n$ and $m \geq 2$.

(i) If $p$ is any prime not dividing $m$, then

$$
p_{m}^{*}: H^{*}\left(W_{n, k ; m} ; \mathbb{Z}_{p}\right) \cong H^{*}\left(W_{n, k} ; \mathbb{Z}_{p}\right)=\Lambda_{\mathbb{Z}_{p}}\left(v_{2 n-2 k+1}, \ldots, v_{2 n-1}\right)
$$

is an isomorphism of algebras.

(ii) If $p$ is a an odd prime that divides $m$, then

$$
\begin{aligned}
H^{*}\left(W_{n, k ; m} ; \mathbb{Z}_{p}\right) & \cong H^{*}\left(\mathbb{S}^{1} ; \mathbb{Z}_{p}\right) \otimes H^{*}\left(P W_{n, k} ; \mathbb{Z}_{p}\right) \\
& \cong \mathbb{Z}_{p}\left[y_{2}\right] /\left\langle y_{2}^{N^{\prime}}\right\rangle \otimes \Lambda_{\mathbb{Z}_{p}}\left(y_{1}, y_{2 n-2 k+1}, y_{2 n-2 k+3}, \ldots, \hat{y}_{N-1}, \ldots, y_{2 n-1}\right)
\end{aligned}
$$

where $N, N^{\prime}$ are as defined above. (As usual, ^stands for omission of the variable.) Also $y_{2}=c_{1}\left(\xi_{n, k ; m}\right) \bmod p$. (iii)(a) Suppose $m \equiv 2 \bmod 4$. Then

$H^{*}\left(W_{n, k ; m} ; \mathbb{Z}_{2}\right)=\mathbb{Z}_{2}\left[y_{1}\right] /\left\langle y_{1}^{N}\right\rangle \otimes \Lambda_{\mathbb{Z}_{2}}\left(y_{2 n-2 k+1}, y_{2 n-2 k+3}, \ldots, \hat{y}_{N-1}, \ldots, y_{2 n-1}\right)$.

(b) Suppose that $m \equiv 0 \bmod 4$. Then

$H^{*}\left(W_{n, k ; m} ; \mathbb{Z}_{2}\right)=\mathbb{Z}_{2}\left[y_{2}\right] /\left\langle y_{2}^{N^{\prime}}\right\rangle \otimes \Lambda_{\mathbb{Z}_{2}}\left(y_{1}, y_{2 n-2 k+1}, y_{2 n-2 k+3}, \ldots, \hat{y}_{N-1}, \ldots, y_{2 n-1}\right)$,

where $y_{1}^{2}=0$.

Proof. (i) Let $\gamma \in \Gamma_{m}$. Recall that $p_{m}: W_{n, k} \longrightarrow W_{n, k ; m}$ is the covering projection with deck transformation group $\Gamma_{m}$. The covering map $\gamma: W_{n, k} \longrightarrow$ $W_{n, k}$ is homotopic to the identity since $\gamma \in U(n)$ and $U(n)$ is connected. It follows that $\Gamma_{m}$ acts trivially on the cohomology groups of $W_{n, k}$. Since $p$ does not divide $m, p_{m}^{*}: H^{*}\left(W_{n, k ; m} ; \mathbb{Z}_{p}\right) \longrightarrow H^{*}\left(W_{n, k} ; \mathbb{Z}_{p}\right)^{\Gamma_{m}}=H^{*}\left(W_{n, k} ; \mathbb{Z}_{p}\right)$ is an isomorphism. One knows that $H^{*}\left(W_{n, k} ; \mathbb{Z}_{p}\right) \cong \Lambda_{\mathbb{Z}_{p}}\left(y_{2 n-k+1}, \ldots, y_{2 n-1}\right)$ (see 4 , Proposition 9.1]).

(ii) By definition, $\xi_{n, k ; m}=\pi_{m}^{*}\left(\zeta_{n, k}\right)$ where $\pi_{m}: W_{n, k ; m} \longrightarrow P W_{n, k}$ is the projection of the principal $U(1) / \Gamma_{m} \cong \mathbb{S}^{1}$-bundle. (See $\S 2$.) Let $y_{2}=$ 
$c_{1}\left(\zeta_{n, k}\right) \in H^{2}\left(P W_{n, k} ; \mathbb{Z}_{p}\right)$. We apply the Serre spectral sequence with $\mathbb{Z}_{p^{-}}$ coefficients to the principal $\mathbb{S}^{1}$-bundle with projection $\pi_{m}$. The differential $d: E_{2}^{0,1} \longrightarrow E_{2}^{2,0}$ maps the generator of $E_{2}^{0,1} \cong H^{1}\left(\mathbb{S}^{1} ; \mathbb{Z}_{p}\right) \cong \mathbb{Z}_{p}$ to $c_{1}\left(\zeta_{n, k}^{m}\right)=$ $m y_{2} \in H^{2}\left(P W_{n, k} ; \mathbb{Z}_{p}\right) \cong \mathbb{Z}_{p} y_{2}$ by Lemma 2.1 (i). Since $p \mid m$, this differential is zero. It follows that the spectral sequence collapses and we get $H^{*}\left(W_{n, k ; m} ; \mathbb{Z}_{p}\right) \cong$ $H^{*}\left(\mathbb{S}^{1} ; \mathbb{Z}_{p}\right) \otimes H^{*}\left(P W_{n, k} ; \mathbb{Z}_{p}\right)$. Note that $y_{1}^{2}=0$ as $p$ is odd. The rest of the statement follows from the description of the $\mathbb{Z}_{p^{-}}$-cohomology of $P W_{n, k}$ due to Astey, Gitler, Micha and Pastor 2.

(iii) We proceed as in (ii) and obtain that $H^{*}\left(W_{n, k ; m} ; \mathbb{Z}_{2}\right) \cong H^{*}\left(\mathbb{S}^{1} ; \mathbb{Z}_{2}\right) \otimes$ $H^{*}\left(P W_{n, k} ; \mathbb{Z}_{2}\right)$ as an $H^{*}\left(P W_{n, k} ; \mathbb{Z}_{2}\right)$-module. Denoting by $\nu$ the real line bundle associated to the double cover $f: W_{n, k ; l} \longrightarrow W_{n, k ; m}$ where $m=2 l$ we have $w_{1}(\nu)=: y_{1}$ is the generator of $H^{1}\left(W_{n, k ; m} ; \mathbb{Z}_{2}\right)$. Also, $\nu \otimes_{\mathbb{R}} \mathbb{C}$ is evidently an element of order 2 in $\operatorname{Pic}\left(W_{n, k ; m}\right)$ and hence $\nu \otimes_{\mathbb{R}} \mathbb{C} \cong \xi_{n, k ; m}^{l}$ by Lemma 2.2. It follows that $y_{1}^{2}=c_{1}(\nu \otimes \mathbb{C})=l c_{1}\left(\xi_{n, k ; m}\right)=l y_{2}$. Hence $y_{1}^{2}=0$ if $l$ is even, and $y_{1}^{2}=y_{2}$ if $l$ is odd.

Finally, write $d=\operatorname{dim} W_{n, k ; m}$. Then $H^{d-1}\left(P W_{n, k} ; \mathbb{Z}_{2}\right) \otimes \mathbb{Z}_{2} y_{1} \cong H^{d}\left(W_{n, k ; m} ;\right.$ $\left.\mathbb{Z}_{2}\right) \cong \mathbb{Z}_{2}$. Therefore $y_{1} y_{2}^{N^{\prime}-1} y_{2 n-2 k+1} \ldots \hat{y}_{N-1} \ldots y_{2 n-1}$ generates $H^{d}\left(W_{n, k ; m} ; \mathbb{Z}_{2}\right)$ $\cong \mathbb{Z}_{2}$. Using this, and the property that square-free monomials in $y_{2 n-2 k+1}$, $\ldots, \hat{y}_{N-1}, \ldots, y_{2 n-1}$ are linearly independent, it follows that the same property holds for $y_{1}, y_{2 n-2 k+1}, \ldots, \hat{y}_{N-1}, \ldots, y_{2 n-1}$. This completes the proof.

We now turn to the integral cohomology of $W_{n, k ; m}$. It is easily seen that $H^{2}\left(W_{n, k ; m} ; \mathbb{Z}\right) \cong \mathbb{Z}_{m}$ generated by $y_{2}=c_{1}\left(\xi_{n, k ; m}\right)$. We are mainly interested in the height of $y_{2}$. Recall that the height of $0 \neq y \in H^{q}(X ; R)$ is the largest positive integer $h$ such that $y^{h} \neq 0$. In view of the fact that the complex Stiefel manifold $W_{n, k}$ is $2(n-k)$-connected, we see that the $2(n-k)$-skeleton of $W_{n, k ; m}$ with respect to any $\mathrm{CW}$-structure may be regarded as the $2(n-$ $k$ )-skeleton of the infinite lens space $L^{\infty}(m)$ with fundamental group $\mathbb{Z}_{m}$. So $H^{q}\left(W_{n, k ; m} ; \mathbb{Z}\right) \cong H^{q}\left(L^{\infty}(m) ; \mathbb{Z}\right) \cong H^{q}\left(\mathbb{Z}_{m} ; \mathbb{Z}\right)$ for $q<2(n-k)$. It is well-known that $H^{*}\left(L^{\infty}\left(\mathbb{Z}_{m}\right) ; \mathbb{Z}\right) \cong \mathbb{Z}\left[y_{2}\right] /\left\langle m y_{2}\right\rangle ;$ see $[6]$. It follows that $H^{q}\left(W_{n, k ; m} ; \mathbb{Z}\right) \cong$ $\mathbb{Z}_{m} y^{r}$ where $q=2 r<2 n-2 k$. However, the following theorem gives the precise value of the height. 
Definition 3.2. Fix integers $n, k, m$ such that $m>1$ and $1 \leq k<n$. We define $m_{r}:=m$ if $r \leq n-k$ and $m_{r}:=\operatorname{gcd}\left\{m,\left(\begin{array}{c}n \\ j\end{array}\right) ; n-k<j \leq r\right\}$ if $n-k<r \leq n$.

The integral cohomology ring of lens spaces is well-known. We shall now establish the following

Theorem 3.3. With the above notations, the (additive) order of $y_{2}^{r} \in H^{2 r}\left(W_{n, k ; m} ; \mathbb{Z}\right)$ is $m_{r}$ for $1 \leq r \leq n$. In particular the height of $y_{2} \in H^{2}\left(W_{n, k ; m} ; \mathbb{Z}\right) \cong \mathbb{Z}_{m}$ is the largest integer $h, n-k<h \leq n$, such that $m_{h}>1$.

Proof. By our observation above, we need only consider the case $r>n-k$.

Let $E$ be a contractible $\mathrm{CW}$ complex on which $\Gamma_{m}$ acts freely so that the quotient $E / \Gamma_{m}=K\left(\Gamma_{m}, 1\right)$ has the same homotopy type as the infinite lens space $L^{\infty}(m)$. Then the fibre product $W^{\prime}:=E \times_{\Gamma_{m}} W_{n, k}$ fibres over $W_{n, k ; m}$ with fibre $E$. In particular, $W^{\prime}$ has the same homotopy type as $W_{n, k ; m}$. Also one has a fibre bundle with fibre $W_{n, k}$ with projection $W^{\prime} \longrightarrow K\left(\Gamma_{m}, 1\right)$. We choose $E$ conveniently so that it is easier to determine the differential in the Serre spectral sequence associated to the $W_{n, k}$-bundle over $K\left(\Gamma_{m}, 1\right)$.

Let $E:=W_{\infty, n}=\bigcup_{r>n} W_{r, n}$ be the space of all unitary $n$-frames in $\mathbb{C}^{\infty}=$ $\bigcup_{r>1} \mathbb{C}^{r}$. An element of $\mathbb{C}^{\infty}$ is viewed as a column vector whose entries are eventually 0 . The space $E$ is contractible since the inclusion $W_{r, n} \subset W_{r+1, n}$ is $2(r-n)$-connected for all $r>n$.

The group $\Gamma_{m}$ acts on $\mathbb{C}^{\infty}$ via scalar multiplication and hence one has the diagonal action of $\Gamma_{m}$ on $W_{\infty, n}$. The quotient $W_{\infty, n} / \Gamma_{m}=: L$ has the homotopy type of the infinite lens space $L^{\infty}(m)$. The image of $\mathbf{v}=\left(v_{1}, \ldots, v_{n}\right) \in W_{\infty, n}$ in $L$ under the quotient map $q: W^{\prime} \longrightarrow L$ will be denoted $\left[v_{1}, \ldots, v_{n}\right]_{m}$ or $[\mathbf{v}]$. The $n$-dimensional complex vector space $\mathbb{C} v_{1}+\ldots+\mathbb{C} v_{n}$ will be denoted $\langle\mathbf{v}\rangle$. Denote by $W_{k}(V)$ the space of all unitary $k$-frames in the complex vector space $V \subset \mathbb{C}^{\infty}$.

Let $W^{\prime}:=\left\{\left([\mathbf{v}]_{m} ; u_{1}, \ldots, u_{k}\right) \mid \mathbf{v} \in W_{\infty, n},\left(u_{1}, \ldots, u_{k}\right) \in W_{k}(\langle v\rangle)\right\}$. The space $W^{\prime}$ is just the $W_{n, k}$-bundle over $L$ associated to the $n$-plane bundle $n \gamma$ where $\gamma$ is the complex line bundle associated to the character $\pi_{1}(L)=\Gamma_{m} \subset \mathbb{S}^{1}$. Let $\widetilde{f}: W^{\prime} \longrightarrow E / U(n-k)=B U(n-k)$ be defined as $\widetilde{f}\left([\mathbf{v}]_{m} ; u_{1}, \ldots, u_{k}\right)=$ $\left(\langle\mathbf{v}\rangle ; u_{1}, \ldots, u_{k}\right)$. We let $f: L \longrightarrow E / U(n)=B U(n)$ be the map $[\mathbf{v}]_{m} \mapsto\langle\mathbf{v}\rangle$. 
One has the projection $\pi: B U(n-k) \longrightarrow B U(n)$, defined as $\left(\langle\mathbf{u}\rangle ; u_{1}, \ldots, u_{k}\right) \mapsto$ $\langle\mathbf{u}\rangle$, of a fibre bundle with fibre $W_{n, k}$. This is just the projection of the $W_{n, k^{-}}$ bundle associated to the universal $n$-plane bundle $\gamma_{\infty, n}$. Clearly $\pi \circ \widetilde{f}=f \circ q$ and $f^{*}\left(\gamma_{\infty, n}\right) \cong n \gamma$. Thus the following diagram commutes:

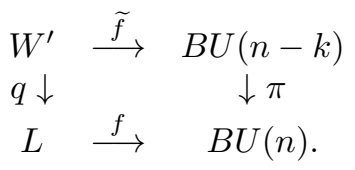

The $W_{n, k}$-bundle $W^{\prime} \longrightarrow L$ is the pull-back of the bundle $B U(n-k) \longrightarrow B U(n)$. In particular the former bundle is $\mathbb{Z}$-orientable. We consider the Serre spectral sequence of the $W_{n, k}$-bundle $q: W^{\prime} \longrightarrow L$ which converges to $H^{*}\left(W^{\prime} ; \mathbb{Z}\right) \cong$ $H^{*}\left(W_{n, k ; m} ; \mathbb{Z}\right)$. We have $E_{2}^{p, q}=H^{p}\left(L ; H^{q}\left(W_{n, k} ; \mathbb{Z}\right)\right)=H^{p}\left(L^{\infty}(m) ; \mathbb{Z}\right) \otimes H^{q}\left(W_{n, k} ; \mathbb{Z}\right)$ since $H^{*}\left(W_{n, k} ; \mathbb{Z}\right)=\Lambda_{\mathbb{Z}}\left(y_{2 n-2 k+1}, \ldots, y_{2 n-1}\right)$ is free abelian. It is well-known that $H^{*}\left(L^{\infty}(m) ; \mathbb{Z}\right) \cong \mathbb{Z}\left[y_{2}\right] /\left\langle m y_{2}\right\rangle$. By comparing the Serre spectral sequence of $\pi: B U(n-k) \longrightarrow B U(n)$, we see that the cohomology classes $y_{2 n-2 k+2 j-1}, 1 \leq$ $j \leq k$, are transgressive. Indeed $\tau\left(y_{2 n-2 k+2 j-1}\right)=c_{2 n-2 k+2 j}(n \gamma) \in H^{*}(L ; \mathbb{Z}), 1 \leq$ $j \leq k$. That is $\tau\left(y_{2 n-2 k+2 j-1}\right)=\left(\begin{array}{c}n \\ k-j\end{array}\right) y_{2}^{n-k+j}$. It follows that $\left(\begin{array}{c}n \\ j\end{array}\right) y_{2}^{r}=0$ in $H^{2 r}\left(W^{\prime} ; \mathbb{Z}\right)$ for $n-k<j \leq r$ and so, since $m y_{2}=0$, we see that the order of $y_{2}^{r} \in H^{2 n-2 k+2 j}\left(W^{\prime} ; \mathbb{Z}\right)$ equals $m_{r}$. In particular, the height $h$ of $y_{2}$ is as stated in the theorem.

A complete description of the ring structure of $H^{*}\left(W_{n, k ; m} ; \mathbb{Z}\right)$ appears to be more intricate. However, it is clear from the above proof that the torsion subgroup in $H^{*}\left(W_{n, k ; m} ; \mathbb{Z}\right)$ is generated by the $y_{2}^{j}, 1 \leq j<h$. Also, it can be seen readily that there exist classes $v_{2 n-2 k+2 j-1} \in H^{*}\left(W_{n, k ; m} ; \mathbb{Z}\right), 1 \leq j \leq k$, which generate a free abelian group of rank $k$. Furthermore, their reduction mod any prime $p$ not dividing $m$ are the generators of $H^{*}\left(W_{n, k ; m} ; \mathbb{Z}_{p}\right)$ given in Theorem 3.1(i). They arise from the generators of the kernel of the transgression in the spectral sequence in the above proof.

As an application we have the following theorem. We write $p(M)$ (resp. $w(M)$ for the total Pontrjagin class (resp. total Stiefel-Whitney class) of a differentiable manifold $M$. (See [16].) 
Proposition 3.4. Let $2 \leq k \leq n-2$. and let $m \geq 2$. One has $p\left(W_{n, k ; m}\right)=$ $\left(1+y_{2}^{2}\right)^{n k}$ for all $r \geq 1$. The total Stiefel-Whitney class $w\left(W_{n, k ; m}\right)=\left(1+y_{1}^{2}\right)^{n k}$, where it is understood that $y_{1}=0$ when $m$ is odd.

Proof. Consider the complexified tangent bundle $\tau_{\mathbb{C}}:=\tau W_{n, k ; m} \otimes_{\mathbb{R}} \varepsilon_{\mathbb{C}}$. From $(4), \tau_{\mathbb{C}}$ is stably equivalent to the complex vector bundle $n k\left(\xi_{n, k ; m} \oplus \xi_{n, k ; m}^{\vee}\right)$. Therefore $c\left(\tau_{\mathbb{C}}\right)=\left(1+y_{2}\right)^{n k}\left(1-y_{2}\right)^{n k}=\left(1-y_{2}^{2}\right)^{n k}$. It follows that the $j$-th Pontrjagin class $p_{j}\left(W_{n, k ; m}\right)=\left(\begin{array}{c}n k \\ j\end{array}\right) y_{2}^{2 j}$.

Using (4) we get $w\left(W_{n, k ; m}\right)=w\left(\xi_{n, k ; m}\right)^{n k}=\left(1+y_{1}^{2}\right)^{n k}$.

Recall from Theorem 2.4 that $W_{n, n-1 ; m}$ is parallelizable for all $m$. The rest of the $W_{n, k ; m}$ are not stably parallelizable for most values of $m$.

Theorem 3.5. Let $1<k \leq n-2$ and $m \geq 2$. If there exists an $r \geq 1$ such that $\left(\begin{array}{c}n k \\ r\end{array}\right)$ is not divisible by $m_{2 r}$, then $W_{n, k ; m}$ is not stably parallelizable. In particular, if $W_{n, k ; m}$ is stably parallelizable, then $m$ divides $n k$.

Proof. If $\left(\begin{array}{c}n k \\ r\end{array}\right)$ is not divisible by $m_{2 r}$, then $m_{2 r}>1$ and so $h>2 r$. Therefore $p_{r}\left(W_{n, k ; m}\right)=\left(\begin{array}{c}n k \\ r\end{array}\right) y^{2 r} \neq 0$ by Proposition 3.4. It follows that $W_{n, k ; m}$ is not stably parallelizable (cf. [16, Lemma 15.2]).

As for the second assertion, since $h>n-k \geq 2$, one has $y_{2}^{2} \neq 0$. If $W_{n, k ; m}$ is stably parallelizable, then $p_{1}\left(W_{n, k ; m}\right)=n k y_{2}^{2}=0$ and hence $m_{2}=m$ divides $n k$.

Remark 3.6. The above theorem does not settle completely the question of stable parallelizability of $W_{n, k ; m}$. Suppose that $n, k$ are powers of a prime $p$ and $m=p$. Then $h=n$ and $m_{r}=p, \forall r<n$. In this case, $p_{j}\left(W_{n, k ; m}\right)=$ $0, w_{j}\left(W_{n, k ; m}\right)=0$ for all $j>0$. We remark that in the case of lens spaces $L^{n}(p)$ where $p$ is an odd prime, Kambe [9] has obtained non-immersion results using K-theory calculations. Combined with the work of Adams 1 on the order of the Hopf bundle $\xi_{n, 1 ; 2}$, one obtains that for a fixed $n$, for all but finitely many $m>1$, the lens spaces are not stably parallelizable. 
Acknowledgment: We thank Július Korbaš for a careful reading of an earlier version of this paper and for his valuable comments. Also we thank the referees for their valuable comments and suggestions. We owe Remark 2.6 and an improved Theorem 2.4(i) to one of them. The first named author thanks the Institute of Mathematical Sciences, Chennai, for its hospitality where this work was carried out.

\section{REFERENCES}

[1] ADAMS, J. F.: Vector fields on spheres, Ann. Math. 75, (1962), 603-632.

[2] ASTEY, L.- GITLER, S.- MICHA, E. -PASTOR, G: Cohomology of complex projective Stiefel manifolds, Canad. J. Math. 51, (1999), 897-914.

[3] BARUFATTI, N.- HACON, D - LAM, K.-Y.- SANKARAN, P.-ZVENGROWSKI, P.: The order of real line bundles, Bol. Soc. Mat. Mexicana 10 (2004), 149-158.

[4] BOREL, A: Sur la cohomologie des espaces fibrés principaux et des espaces homogènes de groupes de Lie compacts, Ann. Math. 57, (1953), 115-207.

[5] GITLER, S.-HANDEL, D.: The projective Stiefel manifolds-I, Topology 7, (1968), 3946.

[6] HATCHER, A.: Algebraic topology, Cambridge University Press, 2002.

[7] HUSEMOLLER, D. Fibre bundles Third Edition, Grad. Texts in Math. 20, SpringerVerlag, N.Y. 1994

[8] IWATA, K.: Span of lens spaces, Proc. Amer. Math. Soc. 26, (1970), 687-688.

[9] KAMBE, T.: The structure of $K_{\Lambda}$-rings of the lens space and their applications J. Math. Soc. Japan 18, (1966), 135-146.

[10] KORBAS̆, J.-ZVENGROWSKI, P.: The vector field problem: a survey with emphasis on specific manifolds, Exposition. Math. 12, (1994), 3-20.

[11] KORBAŠ, J.-ZVENGROWSKI, P.: On sectioning tangent bundles and other vector bundles, Rend. Circ. Mat. Palermo Suppl. 96, (1996), 85-104.

[12] KOSCHORKE, U.: Vector fields and other vector bundle morphisms-a singularity approach, Lecture Notes in Mathematics, 847, Springer, Berlin, 1981.

[13] LAM, K.-Y.: A formula for the tangent bundle of flag manifolds and related manifolds, Trans. Amer. Math. Soc. 213, (1975), 305-314.

[14] LAM, K.-Y.: Sectioning vector bundles over real projective spaces, Quart. J. Math. 23, (1972), 97-106.

[15] LAM, K.-Y.-RANDALL, D.: Periodicity of the geometric dimension for real projective spaces, In: Algebraic Topology: New Trends in Localization and Periodicity, Prog. Math. 136, (1996), 223-235. Brikhäuser, Basel.

[16] MILNOR, J. W.; STASHEFF, J. D.: Characteristic classes, Annals of Mathematics Studies, 76, Princeton University Press, Princeton, N. J. 1974.

[17] WANG, H.-C.: Closed manifolds with homogeneous complex structure, Amer. J. Math. 76, (1954), 1-32.

[18] YOSHIDA, T.: A remark on vector fields on lens spaces, J. Sci. Hiroshima Univ. Ser. A-I Math. 31, (1967), 13-15.

* Tata Institute of Fundamental Research, Mumbai, india.

E-mail address: shilpa@math.tifr.res.in 
** Institute of Mathematical Sciences, Chennai, INDiA

E-mail address: sankaran@imsc.res.in 